\title{
Racial/Ethnic Preferences, Sex Preferences, and Perceived Discrimination Related to End-of-Life Care
}

\author{
Sonia A. Duffy, PhD, RN, ${ }^{* \dagger}$ Frances C. Jackson, PhD, RN, ${ }^{\mathcal{S}}$ Stephanie M. Schim, PhD, RN," \\ David L. Ronis, PhD, ${ }^{* \ddagger}$ and Karen E. Fowler, MPH*
}

This study investigated racial/ethnic preferences, sex preferences, and perceived discrimination related to end-of-life care. Ten focus groups and a follow-up survey were conducted to obtain in-depth information on end-of-life preferences across five racial/ethnic groups in Michigan stratified by sex. There were 73 focus group participants, including Arab Muslims, Arab Christians, Hispanics, blacks, and whites. The mean age \pm standard deviation was $67 \pm 8.5$ (range 50-83). A focus group screener was used to recruit participants. A moderator discussion guide was used to guide the focus groups. A take-home questionnaire asked about demographic information and end-of-life issues.

Arab Americans were in favor of making peace on earth and were against assisted suicide, extending life artificially, nursing homes, and telling the patient "bad news." Hispanic and black women were against assisted suicide and in favor of extending life, whereas the men in these groups felt the opposite. Hispanic women spoke of not wanting a feeding tube and would consider alternative medicine. Blacks were least opposed to nursing homes. For whites, it was important to have choices. When asked about discrimination related to end-of-life care, Muslim women spoke of cultural barriers, blacks spoke of inequities in the past, and whites spoke of age discrimination and abandonment when dying.

From the *Center for Practice Management and Outcomes Research, Ann Arbor Veterans Affairs Hospital, Ann Arbor, Michigan; ${ }^{\dagger}$ Departments of Otolaryngology and Psychiatry, ${ }^{\ddagger}$ School of Nursing, University of Michigan, Ann Arbor, Michigan; ${ }^{\S}$ School of Nursing, Oakland University, Rochester, Michigan and ${ }^{\|}$College of Nursing, Wayne State University, Detroit, Michigan.

This research was jointly funded by the Michigan Department of Community Health, Blue Cross/Blue Shield of Michigan (774.11), and the Department of Veterans Affairs. Data from this manuscript were presented to the Michigan Hospice and Palliative Care Organization, Kalamazoo Michigan, August 2004, and at the Eighth International Congress of Behavioral Medicine, Mainz, Germany, August 27, 2004.

Address correspondence to Sonia A. Duffy, PhD, RN, Ann Arbor Veterans Affairs Hospital $(11 \mathrm{H})$, Center for Practice Management and Outcomes Research, PO Box 130170, Ann Arbor, MI 48113.

E-mail: Sonia.Duffy@med.va.gov

DOI: $10.1111 / \mathrm{j} .1532-5415.2005 .00526 . \mathrm{x}$
As the population becomes more diverse and continues to age, it will be important to provide culturally and sexsensitive end-of-life interventions to increase patient/family satisfaction and allocate resources appropriately. J Am Geriatr Soc 54:150-157, 2006.

Key words: attitude toward death; ethnic groups; population groups

Geveral studies have identified differences in end-of-life $\checkmark$ preferences between different racial/ethnic and sex groups. ${ }^{1-3}$ Most studies have been retrospective, and none have examined discrimination related to end-of-life care. A 2004 National Institutes of Health consensus conference on Improving End-of-Life Care addressed the need to include more minorities in research, use the same measures across racial groups, reduce sex and age disparities, and use multiple methods of data collection. ${ }^{4}$ Given these recommendations, the aging of the population, and the growing numbers of minorities in the United States, this study prospectively identified end-of-life preferences and perceived discrimination of different racial/ethnic and sex groups using a focus group and survey methodology.

\section{RACIAL/ETHNIC INFLUENCES ON END-OF-LIFE PREFERENCES}

Recent literature demonstrates that end-of-life preferences vary significantly between different races/ethnicities, including the reluctance of blacks and Hispanics to use hospice, ${ }^{1,5,6}$ advance directives, and withdraw life-sustaining treatment ${ }^{7}$ and the reluctance of Arab Muslims to unnecessarily prolong life. ${ }^{8}$ In most studies, race/ethnicity remained a significant predictor of end-of-life decisions even when adjusting for demographic and socioeconomic status variables. For example, given equal educational levels, black physicians remained more likely than their non-Hispanic white counterparts to choose aggressive treatment options. ${ }^{9}$ Moreover, level of acculturation (movement toward mainstream American culture) does not alter findings related to cultural influences on end-of-life preferences. ${ }^{10}$ 
Religion/religiosity/spirituality, often associated with personal racial/ethnic affiliation, has been shown to be associated with measures that prolong life, reluctance to withdraw life support, ${ }^{7,10-12}$ and disapproval of assisted suicide and euthanasia. ${ }^{13}$ These findings suggest that, although knowledge and education are modifiable, deeprooted cultural beliefs and values are difficult to influence.

A few studies have been conducted regarding sex and end-of-life care. Women are more likely to experience more pain and be undertreated for pain. Moreover, women are less likely than men to prefer and receive aggressive treatment at the end of life. ${ }^{14-17}$

To better understand end-of-life preferences, 10 focus groups were conducted, stratified by race/ethnicity and sex. The participants were also asked to pretest an end-of-life survey. Institutional review board approval was obtained from the University of Michigan.

\section{METHODS}

\section{Sample}

A professional marketing firm recruited the sample and conducted the groups (in English) at their facility, except for the Arab Muslim women's group, which was conducted at an Islamic Center. A combination of methods to recruit the participants was used, including purchased name lists from RL Polk (a company that provides lists of individuals derived from vehicle registrations and can select people by ethnicity, age, and ZIP code), facility databases, key individuals in the Hispanic and Arab communities who reached out to those communities to provide potential names of people to participate, and directly through Hispanic and Islamic community centers, which asked for volunteers from their membership. Inclusion criteria were self-identification as Arab Muslim, Arab Christian, Hispanic, black, or white; English speaking; aged 50 and older (because minorities often die younger); and no obvious unstable psychiatric or cognitive illness. A focus group screener was used to recruit a purposive sample with a variety of factors that may influence end-of-life preferences, including information on educational level, religion, and previous experiences with death and dying. "Professional respondents" who may lead or monopolize the discussion were excluded if they had participated in a focus group within the previous year. These are standard recruitment procedures for focus groups. ${ }^{18,19}$

\section{Procedures}

Using a moderator discussion guide, the facilitator presented slides, trade-off scenarios, a concept sorting exercise, and issues of discrimination/prejudice for discussion. Some examples of the slides used included someone on a respirator, clergy visiting a patient, and a picture of Dr. Kevorkian. For those concepts that may not be well represented by pictures, verbal scenarios of end-of-life situations were presented (Appendix 1). For example, one scenario was: "You are experiencing extreme pain, but when you take pain medication, you are no longer alert. Would you rather take pain medication and be pain free even if it meant sleeping most of the time?"
Next, participants were asked to participate in a card sort using end-of-life concepts derived from the literature and placed on index cards. Examples of concepts included physician communication, not being a burden, and avoiding nursing home. With each successive focus group, new concepts that emerged were added to the pile of cards. The moderator read each concept out loud and asked participants to sort each card into one of four "hats" that classified the concept as very important, important, neutral/mixed, and not important. If the group could not reach consensus, the concept was placed in the neutral hat. Sorting the concepts into hats allowed the respondent groups to classify the importance of each end-of-life concept for their racial/ethnic and sex group. Last, participants were asked to discuss any discrimination/prejudice that they have experienced related to end-of-life care.

The take-home questionnaire, written in English, asked about demographic information and end-of-life issues. Participants self-identified their ethnicity and race using the U.S. Census Bureau two-tiered question, and cultural affiliation was measured on a 5-point scale by asking respondents how connected they were to their cultural group. End-of-life questions were derived from the literature, end-of-life trade-off scenarios, and previously used questionnaires. ${ }^{10,20}$ The survey results served as participant verification (validity) of the focus group results. ${ }^{21}$

\section{Data Analysis}

The NUDIST software program (Scolari/Sage Publications Software, Newbury Park, CA) was used to support the qualitative analysis. An independent reviewer from the focus group firm transcribed and coded the focus group tapes. Three members of the research team (SAD, FCJ, SMS) independently reviewed the transcripts and identified themes. The researchers compared their findings and identified themes common to each group. The themes from each group were compared and contrasted on a spreadsheet similar to what is shown in Table 1. Quantitative data analysis of the pretest surveys was conducted using the SAS statistical program (SAS Institute, Inc., Cary, NC). Univariate and bivariate analyses were conducted on the survey results to determine differences between groups using means, frequencies, chi-square, and Fisher exact tests. Because the sample size was small and the survey was a pretest, results were given for significant differences $P<.10$. Using triangulation, comparisons were made between the concepts and themes identified in the focus group discussions and survey.

\section{RESULTS}

There were 73 participants in 10 focus groups, with four to nine participants per group. The mean age \pm standard deviation of the entire sample was $67 \pm 8.5$ (range 50-83). Sixty-two of the focus group participants completed the survey, for a return rate of $85 \%$. A summary of the demographic information for each group can be found in Table 2 .

\section{Arabs}

All Arab participants indicated that the family takes care of an individual when they are dying. "Usually it's a son, but the daughters do all the care giving." Being a burden to their 


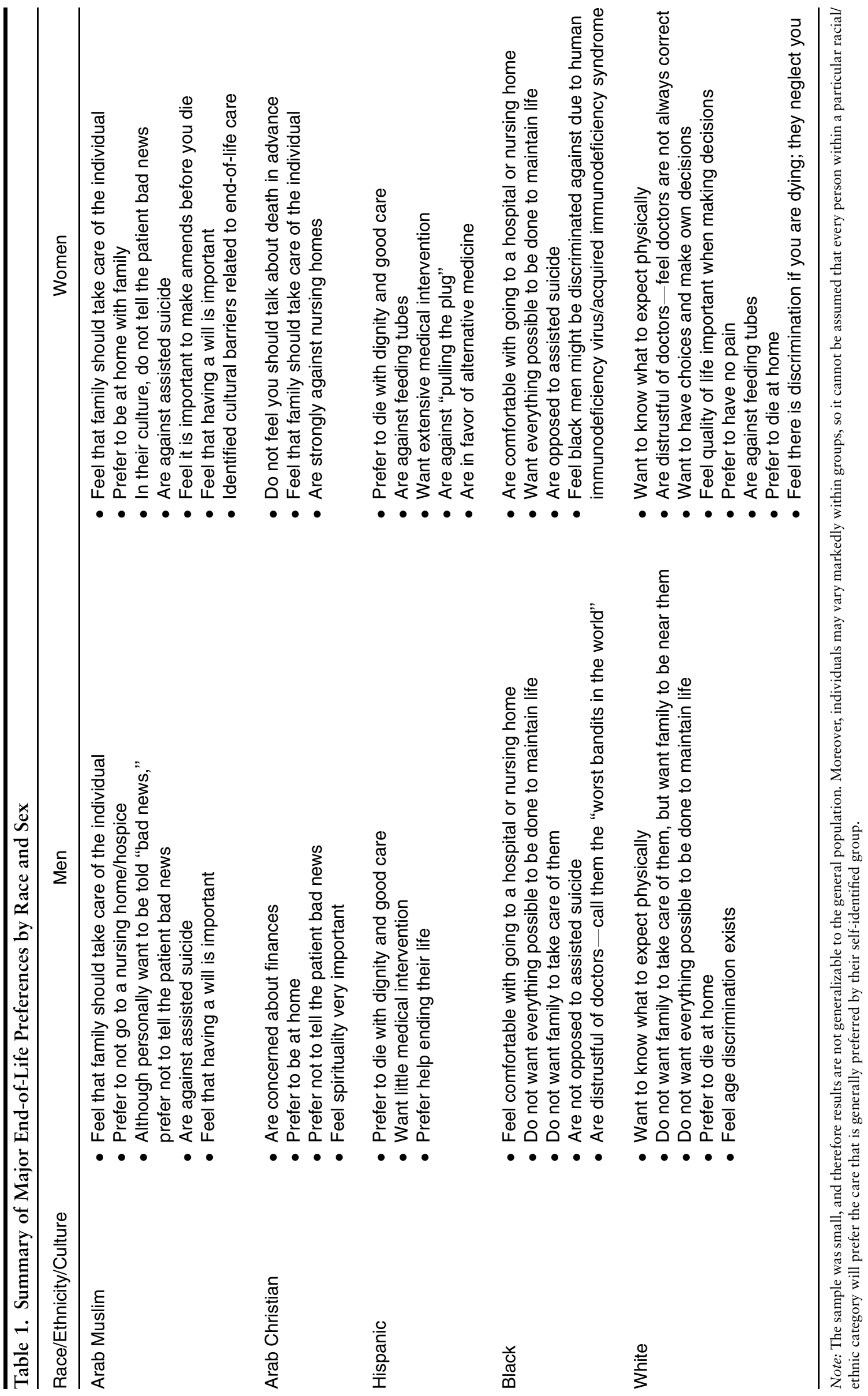


families was not a concern for Arabs. "The whole neighborhood is involved when someone is dying." Arabs "try desperately not to go in a nursing home," which is perceived as the most awful thing that can happen. "There are no nursing homes in Baghdad." In general, Arabs were unfamiliar with the concept of hospice.

Arabs were uncomfortable telling the patient "bad news" and would avoid the word "death" and "cancer." "I said not the whole picture - just half the picture - because she cannot handle it." Alternatively, almost all Arabs said that they personally did want to be told, because it is important to make peace on earth to get into heaven. "The important thing for me is I'm going to look for my deeds ... what I do in my life in front of my children, husband, neighbors, everybody, and I will try to do something before this six months ... before I face God." Arabs were clear that, although "euthanasia is not in our culture," they did not want heroic measures taken. "Never extend life, as there is no value left keeping somebody artificially alive." The survey results indicated that Arabs, more so than others, were significantly more concerned with their accomplishments in life and preferred to have their finances in order before dying (Table 3). Arab men, particularly Arab Christian storekeepers, were concerned with finances. "Regardless of your ethnicity, if you pay, you get the same treatment."

\section{Hispanics}

During the focus groups, Hispanics indicated that dying with "dignity and good care" and "not having to suffer or someone having to change your diapers" was the greatest concern of Hispanics. Hispanics felt that it was important to avoid a nursing home but were receptive to hospitals and hospice. Hispanic women were against feeding tubes. "If I can't eat tortillas no more forget it. Let me go." Hispanic women were the only group that discussed alternative medicine. Survey results indicated that Hispanics were more likely than others to want control their place of death, not to want a feeding tube, and not to have do-not-resuscitate orders (Table 3 ).

Although the survey results indicated that Hispanics preferred assistance with ending their own life, the focus groups indicated marked sex differences. Hispanic women were against "pulling the plug" and wanted extensive medical intervention at the end of life. Unlike Hispanic women, Hispanic men wanted the least amount of intervention at the end of life. "It doesn't make any sense to spend more money to be two months more alive, to make life miserable to others and yourself." Hispanic men were the only group that said they would refuse dialysis and pain medications to stay absolutely alert. Despite being Catholic, Hispanic men more than other groups agreed that they would prefer assisted suicide and wanted to rename assisted suicide to "assisted dying." "I don't think it is suicide if you've already been predetermined and everyone knows that you're going to die."

\section{Blacks}

During focus groups, black men in particular did not feel strongly about the importance of having family and friends take care of them and did not want to burden them. Survey 
Table 3. Selected End-of-Life Preferences by Racial/Ethnic Group $(P<.10)$

\begin{tabular}{|c|c|c|c|c|c|}
\hline \multirow[b]{3}{*}{ End-of-Life Preferences } & \multicolumn{4}{|c|}{ Member of Ethnic Group } & \multirow[b]{3}{*}{$P$-value } \\
\hline & \multicolumn{2}{|c|}{ Yes } & \multicolumn{2}{|c|}{ No } & \\
\hline & \multicolumn{4}{|c|}{$\mathrm{n}(\%)$} & \\
\hline \multicolumn{6}{|c|}{ Arab } \\
\hline \multicolumn{6}{|c|}{ Prefer to remember personal accomplishments } \\
\hline Strongly agree & 9 & (47) & 4 & (10) & \\
\hline Agree to strongly disagree & 10 & (53) & 38 & (90) & $.002^{\dagger}$ \\
\hline \multicolumn{6}{|l|}{ Prefer to have finances in order } \\
\hline Strongly agree & 15 & (79) & 20 & (48) & \\
\hline Agree to strongly disagree & 4 & (21) & 22 & (52) & $.02 *$ \\
\hline \multicolumn{6}{|l|}{ Prefer to die away from home } \\
\hline Neutral/agree & 2 & (11) & 20 & (48) & \\
\hline Disagree & 17 & (89) & 22 & (52) & $.005^{*}$ \\
\hline \multicolumn{6}{|l|}{ Trade-off_resuscitate or DNR } \\
\hline Prefer DNR & 0 & $(0)$ & 20 & (47) & \\
\hline Neutral/prefer resuscitate & 19 & (100) & 23 & (53) & $<.001^{*}$ \\
\hline \multicolumn{6}{|l|}{ How religious are you } \\
\hline Very & 14 & (74) & 17 & (40) & \\
\hline Somewhat to not at all & 5 & (26) & 25 & (60) & $.02 *$ \\
\hline \multicolumn{6}{|c|}{ Hispanic } \\
\hline \multicolumn{6}{|c|}{ Prefer to control time and place of death } \\
\hline Strongly agree & 7 & (54) & 8 & (17) & \\
\hline Agree to strongly disagree & 6 & $(46)$ & 40 & (83) & $.01^{\dagger}$ \\
\hline \multicolumn{6}{|l|}{ Prefer help ending my life } \\
\hline Neutral/agree & 8 & (57) & 12 & (26) & \\
\hline Disagree & 6 & (43) & 35 & (74) & $.049^{\dagger}$ \\
\hline \multicolumn{6}{|l|}{ Trade-off_resuscitate or DNR } \\
\hline Prefer DNR & 9 & (64) & 11 & (23) & \\
\hline Neutral/prefer resuscitate & 5 & (36) & 37 & (77) & $.008^{\dagger}$ \\
\hline \multicolumn{6}{|c|}{ Trade-off-feeding tube or die sooner } \\
\hline Prefer no feeding tube & 12 & (86) & 24 & $(50)$ & \\
\hline Neutral/prefer feeding tube & 2 & (14) & 24 & (50) & $.02^{*}$ \\
\hline \multicolumn{6}{|l|}{ Black } \\
\hline \multicolumn{6}{|l|}{ Prefer to die away from home } \\
\hline Neutral/agree & 10 & (71) & 12 & (26) & \\
\hline Disagree & 4 & (29) & 35 & (74) & $.002 *$ \\
\hline \multicolumn{6}{|l|}{ Prefer to have family present } \\
\hline Strongly agree & 4 & (29) & 31 & (69) & \\
\hline Agree to strongly disagree & 10 & (71) & 14 & (31) & $.007^{*}$ \\
\hline \multicolumn{6}{|l|}{ Trade-off-go home or to ICU } \\
\hline Prefer ICU & 8 & (57) & 2 & (4) & \\
\hline Neutral /prefer home & 6 & $(43)$ & 44 & (96) & $<.001^{*}$ \\
\hline \multicolumn{6}{|l|}{ White } \\
\hline Prefer to know what to expect & & & & & \\
\hline Strongly agree & 12 & (80) & 21 & (45) & \\
\hline Agree to strongly disagree & 3 & (20) & 26 & (55) & $.02 *$ \\
\hline Prefer no pain & & & & & \\
\hline Strongly agree & 10 & (67) & 18 & (38) & \\
\hline Agree to strongly disagree & 5 & (33) & 29 & (62) & $.06 *$ \\
\hline
\end{tabular}

* Chi-square test of association.

${ }^{\dagger}$ Fisher exact test.

$\mathrm{DNR}=$ do not resuscitate; $\mathrm{ICU}=$ intensive care unit.

results indicated that blacks were more likely than others to prefer to die away from home. Blacks preferred staying in the intensive care unit, hospice, or nursing home versus going home and spending time with family (Table 3 ).
Similar to Hispanic women, during focus groups, black women indicated that they were opposed to assisted suicide and pulling the plug and wanted everything done, because "God might not be ready for them yet," and "My doctors 
might have made a mistake.... Anything might turn around." Similar to Hispanic men and unlike black women, black men did not want everything done to maintain life, because "pulling the plug was better than living like a vegetable." Black men preferred pain medications, antibiotics, and dialysis but not life support. Unlike black women, black men were in support of assisted suicide and Dr. Kevorkian, calling him "the man" and equating him with "bravery." Many of the black men were distrustful of doctors (and lawyers) calling them the "worst bandits in the world" and all about "money, money, money."

\section{Whites}

Similar to blacks, during focus groups, whites indicated that they did not feel that it was the family's responsibility to take care of them and were open to hospice and nursing homes, but many preferred to die at home. For whites, it was important to have choices and an advance directive, and they were opposed to extensive measures to extend life. More so than white women, white men wanted cardiopulmonary resuscitation, antibiotics, feeding tubes, and dialysis but not life support. On surveys, whites were more likely to want to know what to expect physically and preferred no pain (Table 3).

\section{Religious Differences}

Arabs were more likely than others to classify themselves as "very religious" $(P=.02)$. Most likely because of the small sample size, there were no significant differences between racial/ethnic groups related to cultural affiliation, although greater religiosity was associated with being more connected to one's cultural group $(P=.007)$. Religiosity was also associated with feeling that it was important to meet with clergy $(P<.001)$, talking about the meaning of life $(P=$ $.10)$, discussing spirituality with the doctor $(P=.004)$, being at peace with God $(P<.001)$, and praying $(P<.001)$. Very religious individuals preferred to remember personal accomplishments $(P=.007)$, to have finances in order $(P=.04)$, to be free of anxiety $(P=.09)$, to feel prepared to die $(P=.04)$, to be listened to $(P=.08)$, and to be comfortable with the nurse $(P=.02)$. Those who were very religious were significantly less likely than others to support ending life, on their own $(P=.01)$ or with assistance $(P=.006)$.

\section{Sex Differences Across Cultures}

Women were found to be more connected to their cultural group than men $(P=.01)$. Women were also more likely than men to want touch $(P=.006)$, feel it was important to pray $(P=.02)$, and prefer to be mentally aware $(P=.08)$. Women were more likely than men to want to resolve unfinished business $(P=.003)$, have living wills $(P=.09)$, and have durable powers of attorney $(P=.02)$.

\section{Discrimination}

When asked an open-ended question about discrimination in end-of-life care, Arab Muslim men said, "This country took care of us more than what we used to be cared for back home." Muslim women felt uncomfortable in the hospital. "I can't sleep or be comfortable. I have to cover and just sit there and expect anybody to walk in any minute." Arab
Christian women indicated that traditional visiting hours are a problem because "Our men are storekeepers and cannot come until late." Although none of the blacks personally experienced discrimination, they did discuss past injustices, including, "A black man created plasma and died, because he could not get it." Black women thought that black men were discriminated against because of acquired immunodeficiency syndrome. White women said that, when you are dying, healthcare providers abandon people. "They put off giving him a water bed figuring he would kick the bucket." White men spoke of age discrimination: "If you do go into an emergency room, ... they will go to the younger person first." An integrated summary of the end of life preferences of each of the groups can be found in Table 1 .

\section{Concepts Important to All}

Concepts important to all groups included being comfortable, physician communication, having responsibilities taken care of, hope and optimism, and honoring spiritual beliefs. Other concepts common to most groups were love and compassion, being cared for, expressing feelings, fixing relationships, saying good-bye, having choices, making plans, not being in pain, and being ready to go.

\section{DISCUSSION}

The reluctance of Arabs to tell the patient bad news has been noted in other studies ${ }^{10}$ and is in direct conflict with Western medicine, which is designed to tell the patient the facts. The Arabs in this study were against telling their family members bad news, but they themselves wanted to know. The withholding of information by the family (when the patient wants to know) can cause a dilemma for healthcare providers. Although healthcare providers are obligated to tell the patient the truth, it may be beneficial to take the time to negotiate with the family and allow them to break the news slowly.

Avoiding a nursing home was crucial for Arabs and Hispanics. This may be a problem for new immigrants to the United States who do not have extended family available. Because Arabs felt strongly that life should not be extended artificially, hospice services may be a more acceptable option than a nursing home, yet most of the Arabs and Hispanics in this study were unfamiliar with hospice services. For minority patients, hospice may be seen as intruding and conflicting with cultural norms. Identifying key community leaders and presentations in churches/mosques can be instrumental to introducing the concept of hospice to minority populations. ${ }^{1}$

Despite their preference for life-sustaining treatment, avoiding a feeding tube was particularly important for Hispanic women because ethnic food and celebration is an integral part of their life. Similarly, one study showed that preference for a feeding tube is stronger in men than women. ${ }^{22}$ However, another study showed that Hispanics were more likely than other races to choose feeding tube as an option. ${ }^{3}$ Although no definite conclusions can be drawn, healthcare providers need to be clear about the lifestyle implications of the end-of-life options.

Although various studies indicate that minorities and men prefer more life-sustaining treatment than whites and women, ${ }^{12,14,16,23}$ in this study, Hispanic and black 
men did not want life-sustaining treatment and approved of assisted suicide. The inconsistency may be because many prior studies did not explore end-of-life preferences by race/ ethnicity and sex. The sex differences within groups could be a problem, because spouses often make decisions regarding end-of-life treatments. Wives are three times as likely to be surrogate decision-makers as husbands. ${ }^{7}$ In general, surrogates often do not accurately reflect the wishes of their loved ones. ${ }^{24}$ Healthcare professionals should encourage couples to discuss end-of-life preferences while they are still able to do so.

Similar to other studies, ${ }^{13}$ this study found an inverse association between religiosity and euthanasia. Unlike other studies, ${ }^{7,11-13}$ no association between religiosity and preferring life-sustaining treatment was found in this study. In fact, Arab Americans in this study, who were the most religious, did not believe in life-sustaining treatment. Prior studies of religiosity and end-of-life care may have not included Arab Americans, who have only recently entered the United States in greater numbers. Differences in end-of-life preferences between different racial/ethnic groups mediated by religiosity suggest the strong need to provide a spiritual assessment and referrals to clergy.

Similar to other studies, 3,25 the black men in this study were the most distrustful of doctors. The respondents' concern over age discrimination is supported by the literature in that do-not-resuscitate orders, less life-sustaining treatment, and more comfort care have been found to be assigned more often to older female patients, even after adjusting for level of illness, functional impairment, comorbidities, and preferences. ${ }^{15,17,26}$ The respondents also noted discrimination related to diagnostic categories (such as acquired immunodeficiency syndrome) and avoidance of dying people.

Healthcare professionals need to keep their personal values in check regarding race/ethnicity/culture, sex, age, and diagnoses. Moreover, healthcare providers should avoid abandoning dying patients and should build trust with patients and their families. Even so, good intentions cannot abolish a history of inequities and mistrust between groups. ${ }^{27}$ The need to educate healthcare providers in cultural sensitivity and recruit more minority healthcare providers has been suggested. ${ }^{28}$ Moreover, healthcare providers should remember that racial/ethnic differences in end-of-life preferences can be used to guide practice and improve cultural sensitivity but do not supersede the need to recognize varying individual preferences. Healthcare providers should also consider the many end-of-life concerns that were important to all groups.

Several important limitations to the study should be noted. This cross-sectional study did not examine changes in end-of-life preferences over time. The focus groups and surveys were in English, and only English-speaking minorities were included in the study. The white groups were ethnically diverse and not naturally stratified by religion. Minority groups such as Asians and American Indians were not included. The sample size was small, which was the trade-off for the rich focus group data that were obtained. In accordance with focus group methodology, the sample was purposively selected to gain in-depth information, but the data are not generalizable to the population. A larger, randomly selected survey that could control for other in- fluential variables (such as religion and socioeconomic status) would be more generalizable. Caution should be taken not to generalize and stereotype individuals, recognizing that individual preferences supersede group norms.

Despite these limitations, the findings from this study can provide insights into the development of culturally and sex-sensitive end-of-life interventions. The provision of culturally competent care can increase patient/family satisfaction. Moreover, providing culturally competent care can increase cost effectiveness by eliminating services that are not acceptable to selected groups and individuals and enhancing those that are effective.

\section{ACKNOWLEDGMENTS}

The authors would like to thank the staff of Market Strategies, Inc., including Fred Manske, Carolyn Holmes, and Kourtney Hill, for their assistance in conducting the focus groups. In addition, the staff would like to thank Ratib Habbal and Amalia McIntyre for their assistance with recruiting minority populations for the focus groups and Carrie Karvonen for providing administrative assistance. Finally, the authors would like to thank the focus group participants who participated in this study for their time and cooperation.

Financial Disclosure(s): The authors received no financial support on this study and have no conflict of interest to report in relation to this research.

Author Contributions: Sonia A. Duffy was the principal investigator and was involved in study concept and design, acquisition of subjects and data, interpretation of data, and preparation of the manuscript. Frances C. Jackson and Stephanie M. Schim were coinvestigators and were involved in study concept and design, acquisition of subjects and data, interpretation of data, and preparation of the manuscript. David L. Ronis was a coinvestigator and was involved in study concept and design, acquisition of data, analysis and interpretation of data, and preparation of the manuscript. Karen E. Fowler was the project coordinator and was involved in study design, acquisition of subjects and data, analysis and interpretation of data, and preparation of the manuscript.

Sponsor's Role: None.

\section{REFERENCES}

1. Noggle BJ. Identifying and meeting needs of ethnic minority patients. Hosp J 1995;10:85-93.

2. Eleazer GP, Hornung CA, Egbert CB et al. The relationship between ethnicity and advance directives in a frail older population. J Am Geriatr Soc 1996; 44:938-943.

3. Caralis PV, Davis B, Wright $\mathrm{K}$ et al. The influence of ethnicity and race on attitudes toward advance directives, life-prolonging treatments, and euthanasia. J Clin Ethics 1993;4:155-165.

4. National Institutes of Health. National Institutes of Health State-of-theScience Conference Statement: Improving End-of-Life Care. Bethesda, MD, December 6-8, 2004 [on-line]. Available at http://consensus.nih.gov/ta/024/ 024EndOfLifepostconfINTRO.htm Accessed April 25, 2005.

5. Gordon AK. Hospice and minorities: A national study of organizational access and practice. Hosp J 1996;11:49-70.

6. Talamantes MA, Lawler WR, Espino DV. Hispanic American elders. Care giving norms surrounding dying and the use of hospice services. Hosp J 1995; 10:35-49.

7. Hornung CA, Eleazer GP, Strothers HS 3rd et al. Ethnicity and decision-makers in a group of frail older people. J Am Geriatr Soc 1998;46:280-286.

8. Sarhill N, LeGrand S, Islambouli R et al. The terminally ill Muslim: Death and dying from the Muslim perspective. Am J Hosp Palliat Care 2001;18:251-255. 
9. Mebane EW, Oman RF, Kroonen LT et al. The influence of physician race, age, and gender on physician attitudes toward advance care directives and preferences for end-of-life decision-making. J Am Geriatr Soc 1999;47:579-591.

10. Blackhall L, Murphy S, Frank G et al. Ethnicity and attitudes toward patient autonomy. JAMA 1995;274:820-825.

11. Burns S. Spiritual care at the end of life. Health Prog 1998;79:45, 46, 49

12. Blackhall LJ, Frank G, Murphy ST et al. Ethnicity and attitudes towards life sustaining technology. Soc Sci Med 1999;48:1779-1789.

13. Cicirelli V. Elders' end-of-life decisions: Implications for hospice care. Hosp J 1997;12:57-72.

14. Bookwala J, Coppola KM, Fagerlin A et al. Gender differences in older adults' preferences for life-sustaining medical treatments and end-of-life values. Death Stud 2001;25:127-149.

15. Johnson MF, Lin M, Mangalik S et al. Patients' perceptions of physicians' recommendations for comfort care differ by patient age and gender. J Gen Intern Med 2000;15:248-255.

16. Carmel S, Mutran E. Preferences for different life-sustaining treatments among elderly persons in Israel. J Gerontol B Psychol Sci Soc Sci 1997;52B:S97-S102.

17. Wenger NS, Pearson ML, Desmond KA et al. Epidemiology of do-not-resuscitate orders. Disparity by age, diagnosis, gender, race, and functional impairment. Arch Intern Med 1995;155:2056-2062.

18. Morgan DL. The Focus Group Guidebook: Focus Group Kit 1. Thousand Oaks, CA: Sage Publications, Inc., 1998.
19. Krueger RA. Developing Questions for Focus Groups: Focus Group Kit 3. Thousand Oaks, CA: Sage Publications, Inc., 1998.

20. Tulsky JA. Understanding What Is Important at the End of Life, 2000 [on-line]. Available at: http://hsrd.durham.med.va.gov/palliative/downloads/finalsurveypatients.doc Accessed March 2001.

21. Krueger RA. Analyzing and Reporting Focus Group Results: Focus Group Kit 6. Thousand Oaks, CA: Sage Publications, Inc., 1998.

22. O'Brien LA, Siegert EA, Grisso JA et al. Tube feeding preferences among nursing home residents. J Gen Intern Med 1997;12:364-371.

23. Hopp FP, Duffy SA. Racial variations in end-of-life care. J Am Geriatr Soc 2000;48:658-663.

24. Hines SC, Glover JJ, Babrow AS et al. Improving advance care planning by accommodating family preferences. J Palliat Med 2001;4:481-489.

25. Jackson F, Schim S, Seely S et al. Barriers to hospice care for African Americans. Problems and solutions. J Hosp Palliat Nurs 2000;2:65-72.

26. Hamel MB, Lynn J, Teno JM et al. Age-related differences in care preferences, treatment decisions, and clinical outcomes of seriously ill hospitalized adults: Lessons from SUPPORT. J Am Geriatr Soc 2000;48:S176-S182.

27. Hallenbeck JL. Intercultural differences and communication at the end of life. Prim Care 2001;28:401-413.

28. Smedley BD, Stith AY, Nelson AR, eds. Unequal Treatment. Confronting Racial and Ethnic Disparities in Health Care. Washington, DC: The National Academies Press, 2003.

\section{Appendix 1}

\section{End-of-Life Trade-Off Scenarios}

Pretend that you have just been diagnosed with a terminal illness and have been told that you are expected to live only 6 more months. During this time, you will be faced with several difficult choices to make about your care. A series of choices will be presented to you. I would like you to tell me what your choices would be and why.

Scenario 1-Dying at Home Versus Being a Burden to Family

You are being discharged from the hospital but require a great deal of personal care. Would you rather go home knowing that your care would be difficult for your family, or would you rather go to a nursing home or hospice?

Scenario 2-Disclosure Versus Nondisclosure of Information to the Patient

You have been diagnosed with a terminal illness, but your doctor has not told you yet. Would you want to be informed and make your own decisions, or would you want your family to be told and be responsible for the decisions?

Scenario 3-Pain Control Versus Being Mentally Alert

You are experiencing extreme pain, but when you take pain medication, you are no longer alert. Would you rather take pain medication and be pain free even if it meant sleeping most of the time?

Scenario 4-Antibiotics Versus Extending the Dying Process

Sometimes antibiotics are given to patients to fight infection. Would you want to take antibiotics to extend your life knowing that it would prolong the dying process?

Scenario 5-Intensive Care Even If No Hope of Recovery

You have the chance to stay in intensive care and have everything done to prolong your life as long as possible or go home and spend your remaining days spending time with your family. Which would you rather do?

Scenario 6-Cardiopulmonary Resuscitation Versus No Chance of Recovery.

You have an unexpected allergic reaction to a medication. The reaction causes your heart to stop. Would you want to be resuscitated even though you have only 6 months left to live?

Scenario 7-Respirator (Short or Long Term)

You are having trouble breathing and have the choice of spending your remaining days on a respirator or dying within several days. Which would you rather do?

Scenario 8-Feeding Tube (Short or Long Term)

You are no longer able to eat. Your doctor offers to put a feeding tube into your stomach. Would you want to be kept alive with a feeding tube and prolong the dying process or die within a couple of weeks without a feeding tube?

Scenario 9-Dialysis (Short or Long Term)

You have kidney disease, which requires you to go to the hospital three times a week for half a day to have your blood cleaned by dialysis. Would you want to continue this even though you only have 6 months to live?

Scenario 10-Withdrawing Treatment Versus Prolonging Suffering

You are in a great deal of pain, and your pain medication is not working. You have been placed on a respirator. Would you want to remain on a respirator and prolong your life temporarily, or would you want life support removed? 